\title{
Click chemistry
}

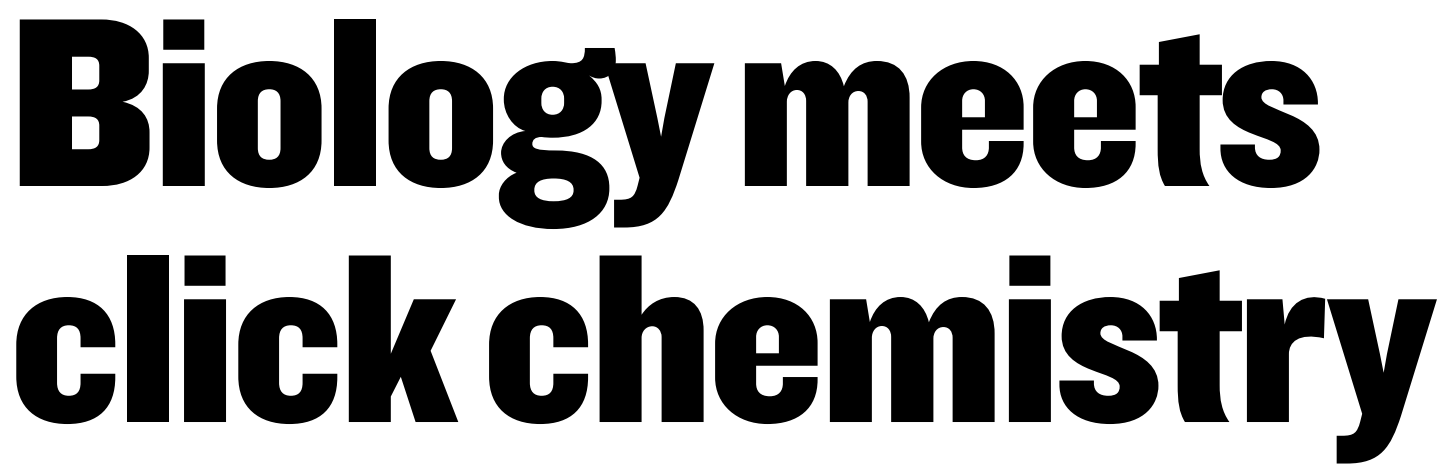

A decades-old reaction that has become the poster boy for the field of click chemistry' is now expanding into biology, as Hayley Birch discovers

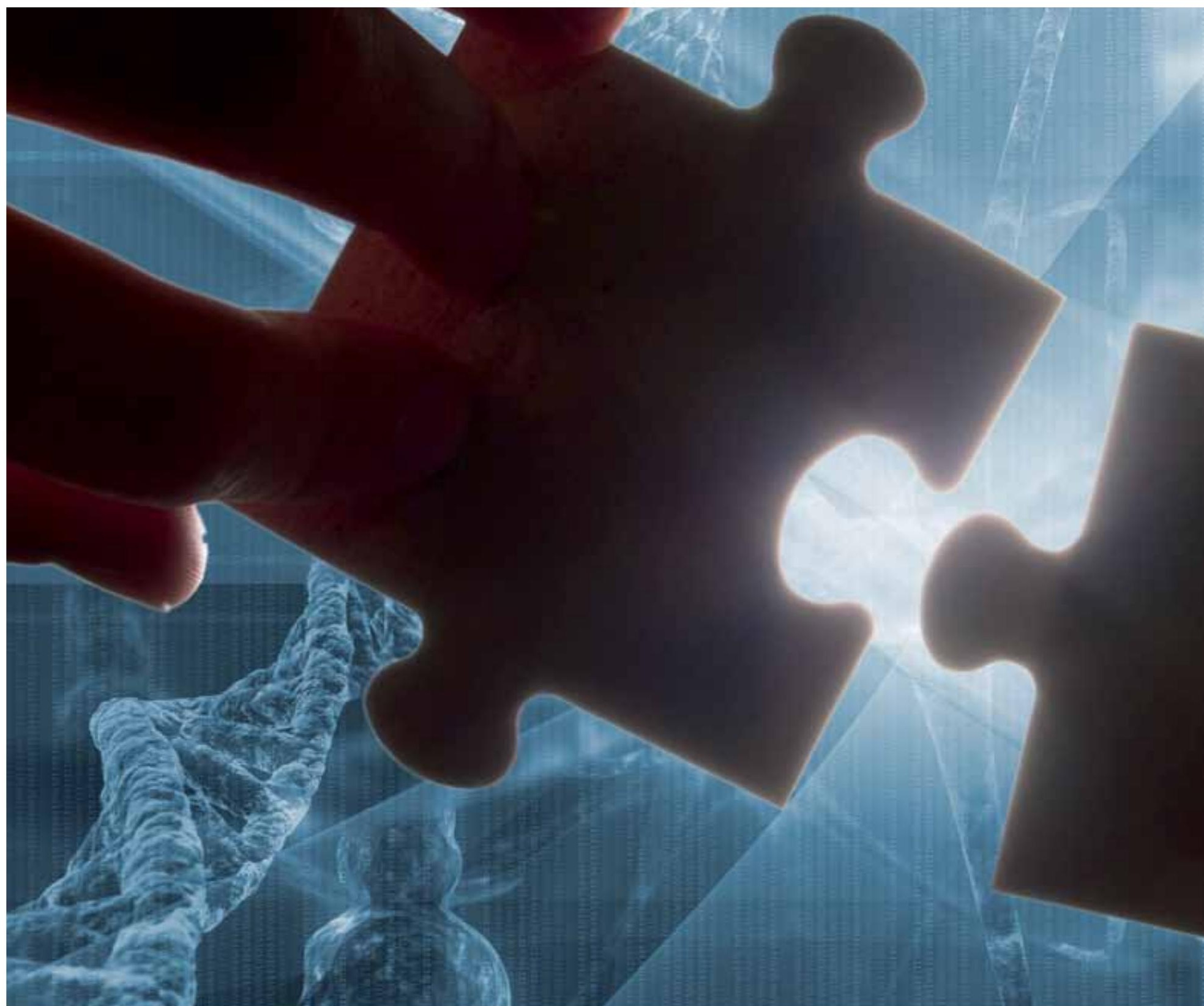


Unlike study of biochemical processes, tissue regeneration requires live cells and DeForest's team was looking for a method that would be compatible with their differentiating stem cells.

\section{Copper-free clicks}

In 2007, a paper by Jeremy Baskin at the University of California, Berkeley, US, and colleagues presented a solution to the problem - copper-free click chemistry performed in live cells. ${ }^{2}$ Based on the classic Huisgen cycloaddition, Baskin's revamped version placed the alkyne group in a 'strained' cyclooctyne ring, adding fluorine atoms to withdraw electrons. The idea was to put tension on the click components to make them more reactive and to drive the reaction

In 2008, Baskin and colleagues went on to prove that the method worked in live animals when they used it to watch cells and tissues forming in zebrafish embryos (Chemistry World, June 2008, p31). They achieved this by snapping fluorescent tags onto sugars in the cell membranes of the fish - the sugars acted as indicators of developmental processes.

The ring strain method was soon adopted by DeForest's lab. Last without the need for a catalyst. year, DeForest was lead author of a

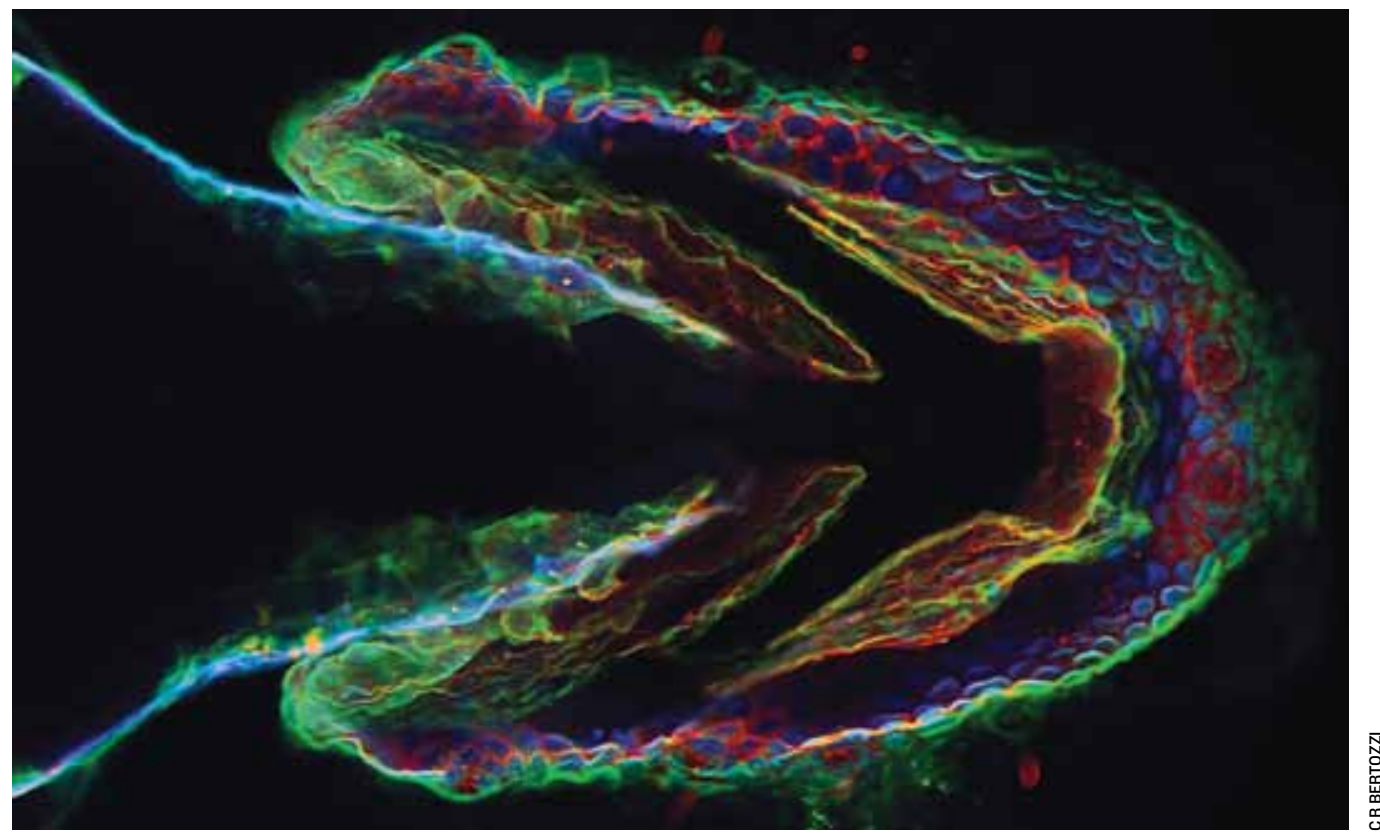

study published in Nature Materials describing a method for creating hydrogels using copper-free click chemistry. ${ }^{3}$ 'We end-functionalise polymers and synthetic peptides with these chemical moieties and then we mix the polymers and the peptides together and they undergo this click reaction which is responsible for forming the hydrogels,' says DeForest.
Sugars, tagged using click chemistry, allow the development of zebrafish to be watched

\section{Leukaemia drugs}

Click chemistry can be used to rapidly assemble a library of hundreds of potential drug candidates. Last year, Shao Yao and colleagues at the National University of Singapore did just that to generate candidates for the treatment of chronic myelogenous leukaemia (CML) ${ }^{5}$ - which affects the white blood cells and is responsible for up to a fifth of all cases of leukaemia. The only known cure is a bone marrow or stem cell transplant.

Several drugs have, however, been approved that can prolong survival, and Yao's team was able to identify a few more potentials from its screens. The approach was based on targeting Abelson tyrosine kinase, an enzyme implicated in CML, and using Huisgen's classic alkyne-azide reaction.

First, as proof of principle, the researchers produced a library of molecules through click reactions between an adenosine diphosphate (ADP)-alkyne and 344 different azides. Then they took imatinib, the first-line therapy for CML patients, and swapped its piperazine moiety for an alkyne, generating a clickable molecule with an already proven drug attached. This was then snapped onto 45 different azides and the products ability to inhibit Abelson tyrosine kinase tested, with some showing a slight improvement over the original imatinib.

According to Yao, his team is already making second and third generations of its candidate drugs. 'Our new generations focus on improving the binding property of the probes, for instance, by using more potent drug templates such as dasatinib instead of imitinib,' he says.

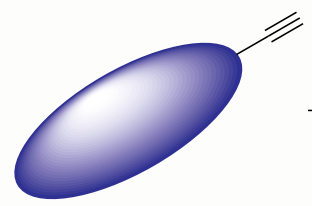

Imatinib-alkyne

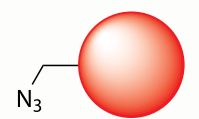

$\mathrm{CuSO}_{4}$

ascorbate

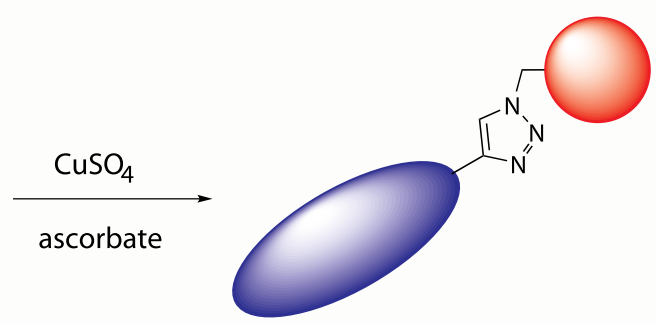

Azides
'And because we've done this with a copper-free click chemistry, we can actually form these hydrogels in the presence of cells without sacrificing viability.' The advantage over traditional methods, he says, is that the click reaction is high yielding, quick and doesn't form any by-product - if it did, this could also potentially be toxic to cells.

\section{Patterning hydrogels}

But that's not all. A second click reaction is used to 'pattern' biological functionality into the 3D network. Straying away from the gold standard Huisgen's reaction, the team employed a light-induced thiol-ene reaction - the addition of a thiol to an alkene - allowing them to snap any thiol-containing compound of their choice onto the main body of the hydrogel. Thus, by incorporating a thiol into an adhesive molecule and shining a light on specific areas, they were able to pattern in attachment sites for cells and show that the cells did, indeed, attach to them. Few technologies have this level of control, claims DeForest.

The team is now working on being able to pattern in more exotic molecules such as growth factors and peptides that may induce differentiation. 'We envision that we will be able to encapsulate a uniform population of stem cells into our hydrogel and then pattern in different growth factors to induce different lineages,' says DeForest. 'So we will be able to create very complex tissue-like structures of spatially controlled, differentiated stem cells.'

A library of drug candidates was made by altering the existing drug, imatinib, using click chemistry 


\section{Tracking devices}

While DeForest attempts to generate tissues from scratch, click chemistry methods are being developed by neurobiologists to track dividing cells Adrian Meedeniya, a neurobiologist at Griffith University in Brisbane, Australia, has based his work on that of a team at Harvard Medical School, which published a method for detecting DNA synthesis using click chemistry in $2008 .^{4}$ The method involved creating an analogue of one of the nucleosides contained in DNA - uridine - with an all-important alkyne group, which was exposed in the groove of the DNA helix. The researchers 'clicked' this with a fluorescent azide that could be imaged to track DNA synthesis and therefore cell division.

Meedeniya considers this work to be 'seminal'. He has since employed 5-ethynyl-2'-deoxyuridine (Edu), a uridine analogue, to track cell division in embryonic mouse brains. So far though, his methods aren't completely compatible with live cell systems; the next step, he says, will be removing copper from the equation. But Edu does improve significantly on its predecessor Brdu, antibody. As Meedeniya explains, to detect this antibody the DNA has to be unzipped, requiring a fairly harsh protocol and resulting in complete destruction of the cell's molecular profile. In other words, genetic and proteomic information about the cell that could be important later is lost. 'That was our major hurdle, and with Edu and the click chemistry protocol we can conserve the molecular profile,' says Meedeniya. 'We don't have to enable it too much or even unzip the DNA. It's a very quick, easy protocol and a really powerful tool for looking at dividing cells.'

So powerful that Meedinya is working on a series of studies using Edu to try to unravel aspects of in naturally regenerating brain tissue. which is detected by a fluorescent

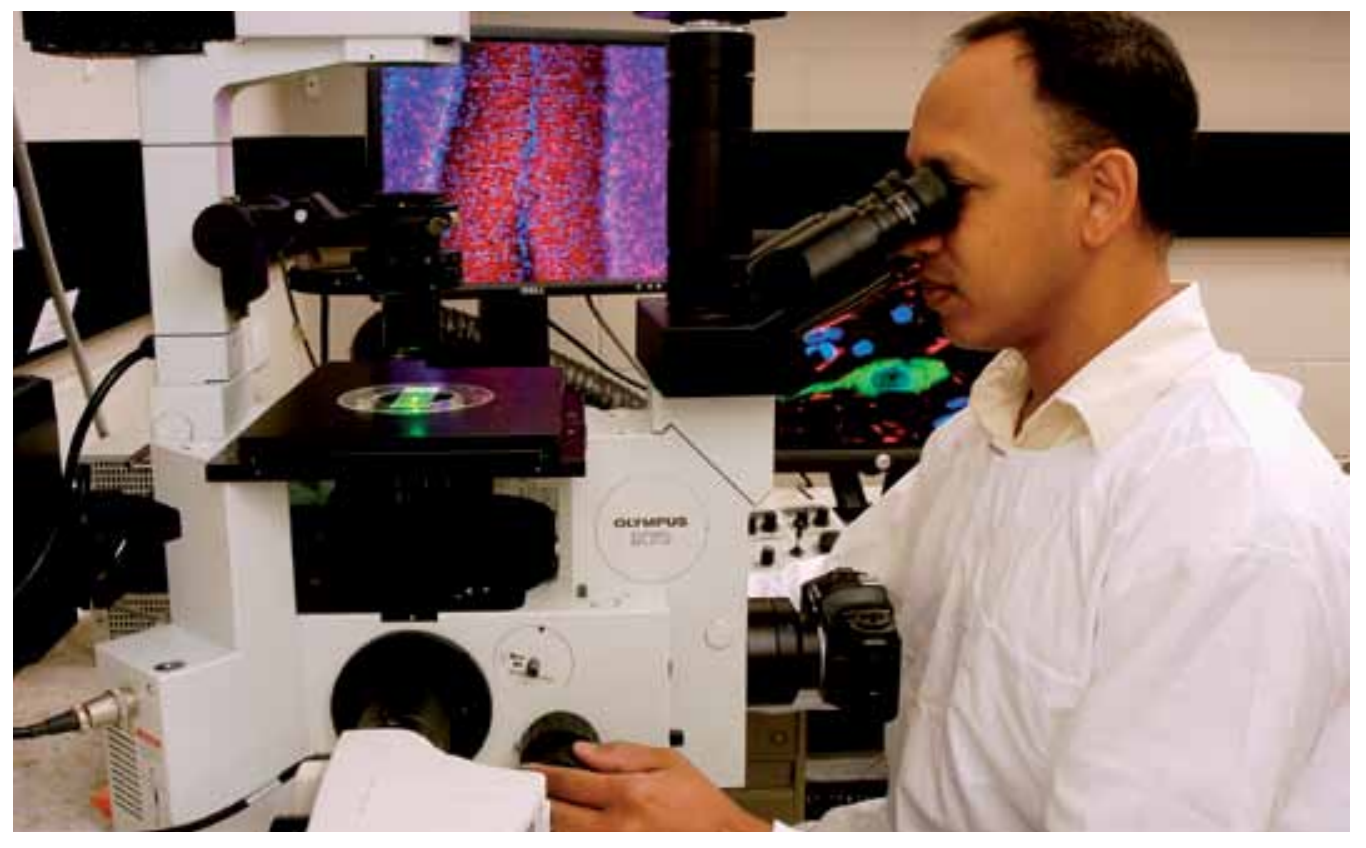

various diseases. Some focus on neurodegenerative disorders, such as Parkinson's, while one is aimed at revealing the mechanisms that regulate cell division in glioblastoma multiforme, the most aggressive form of brain cancer. He is also helping to develop click chemistry products that will function as standard assays and may be invaluable in stem cell products are already marketed by California, US-based biotechnology company, Life Technologies.)

\section{Room for more}

But are too many chemists and biologists still clinging to Huisgen's reaction? It is, after all, celebrating its 50th birthday this year. 'I think there are certainly many other [click] reactions,' says DeForest. 'There's definitely room for pursuing more biologically compatible click reactions, that's for sure.' In theory, he adds, click reactions are supposed to be orthogonal to one other, meaning one reaction can be and cancer research. (Several Click-it
Adrian Meedeniya has used click chemistry to track cell division in mouse brains

Cole DeForest's team uses click chemistry to make hydrogels for culturing cells in 3D
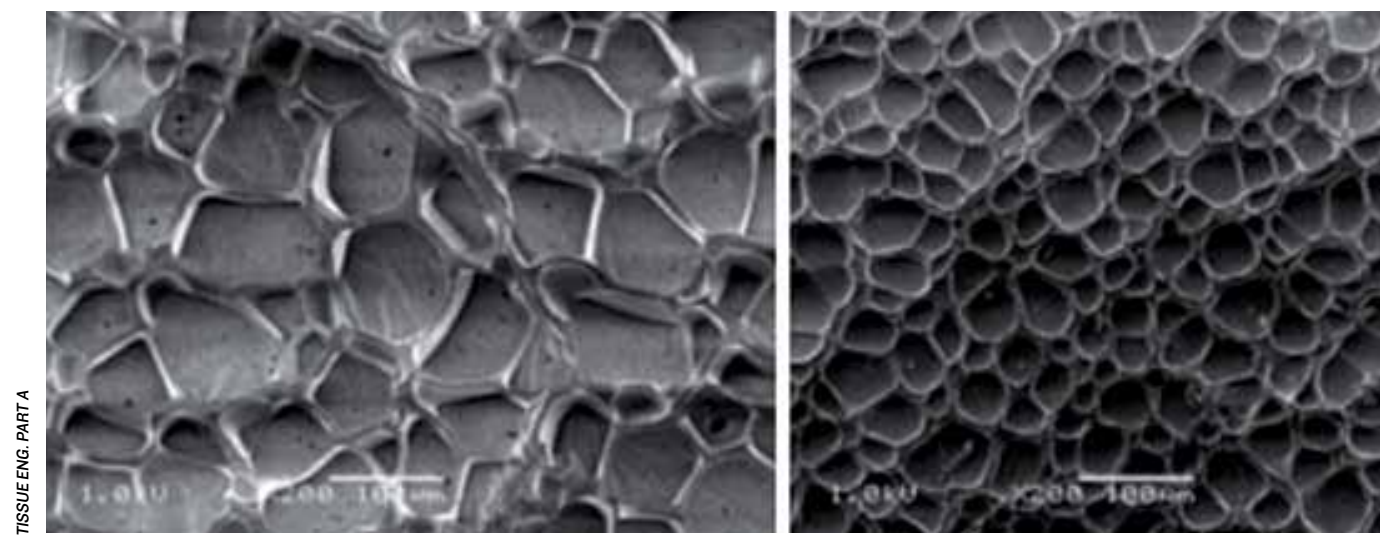

performed in the presence of other functionalities without undergoing side reactions. So discovering more click reactions could mean gaining more control over a system but also providing scope for multiple staining, allowing researchers to track different populations of cells at the same time.

As for Sharpless' original intention of developing easy-to-make drugs, several companies are already rumoured to be working on click products, including Intezyne in Florida. The company is using click methods to modify cancer drugs in such a way that they can be better targeted at tumours. Meanwhile, a group at the National University of Singapore led by Shao Yao has adopted click chemistry techniques to rapidly synthesise inhibitors for enzymes thought to be involved in chronic myelogenous leukemia and to help identify targets for malaria drugs (see box, p38). The growing compatibility of click chemistry with biology suggests its potential is greater than Sharpless, and certainly Huisgen, would ever have guessed.

Hayley Birch is a science writer based in Bristol, UK

\section{References}

$1 \mathrm{H} \mathrm{C}$ Kolb et al, Angew. Chem. Int. Ed., 2001, 40 2004

2 J M Baskin et al, Proc. Natl. Acad. Sci.

USA, 2007, 104, 16793 (DOI: 10.1073/ pnas.0707090104)

3 C A DeForest et al, Nature Mater., 2009, 8, 659 (DOI: 10.1038/nmat2473)

4 A Salic and T J Mitchison, Proc. Natl. Acad. Sci. USA, 2008, 105, 2415 (DOI: 10.1073/ pnas.0712168105)

$5 \mathrm{~K}$ A Kalesh et al, Org. Biomol. Chem., 2009, 7 , 5129 (DOI: 10.1039/b913333j) 